\title{
Dual-sided Mapping During Global Stretch Using a Custom Miniaturized Endocardial Balloon With a Multipurpose Multichannel Acquisition System for Preclinical Electrophysiological Studies
}

\author{
Conrado J. Calvo ${ }^{1,2,5}$, Álvaro Tormos ${ }^{1,5}$, Eduardo Roses ${ }^{1}$, Manuel Zarzoso ${ }^{4}$, Oscar Arias ${ }^{5}$, Antonio \\ Cebrián $^{1}$, S. Jimenez-Serrano ${ }^{1}$, Elena Simarro ${ }^{1}$, José Millet ${ }^{1,5}$, Javier Chorro ${ }^{3,5}$, Antonio Guill ${ }^{1,5}$ \\ ${ }^{1}$ ITACA, Department Electronic Engineering, Universitat Politècnica de València, València, Spain \\ ${ }^{2}$ Departments of Physiology, Medicine ${ }^{3}$ and Physiotherapy ${ }^{4}$, Universitat de València, València, Spain \\ ${ }^{5}$ CiberCV, IIS Incliva-Cardiology Unit, Hospital Clínico Universitario, València, Spain
}

\begin{abstract}
Acute regional myocardial stretch (STR) is known to induce myocardial electrophysiological modifications via mechano-electric feedback (MEF), including abnormal repolarization, premature excitation and increased complexity of activation leading to the initiation, maintenance and acceleration of arrhythmic events. Due to technical limitations little is known about intramural and endo-epicardial (ENDO-EPI) phenomena during these events. Understanding ENDO-EPI modifications produced by STR through MEF may require a dual-sided approach to simultaneously correlate macroscopic surface modifications and their independent or interconnected components. For that purpose, we optically mapped (OM) the EPI LV-surface, and developed a miniaturized multifunctional array balloon (MEAb) for ENDO mapping of small-to-mid sized hearts using a custom-developed scalable FPGA-based multichannel acquisition system (cMCAS). Our aim was two-fold to validate 1) the quality of the sub-endocardial electrograms (EGM) and its association to EPI voltagetransients; and, 2) to study the modifications produced by moderate STR on normal and abnormal activation. ENDO-EPI differences found in restitution properties could be implicated in STR-driven VF-mechanisms.
\end{abstract}

\section{Introduction}

The heart is an inherently mechanosensitive organ. [1] Myocardial stretch either in cells or the whole heart may produce cardiac electrophysiological alterations, rhythm disturbances or, under certain conditions, increase ventricular vulnerability to trigger and/or perpetuate complex arrhythmias. [2] In the clinical setting, STR may appear in different situations including changes in cardiac loading and mechanics, intracardiac myocardial fiber lengthening due to pressure surges or pathologic atrial/ventricular dilatation, changes in regional volume or contractility, procedural device-tissue interactions, or even direct mechanical stimulation. [2,3] This mechanoelectrical feedback, is a mechanism whereby mechanical deformation or alterations in mechanical loading of the heart induces changes in cardiac electrophysiology, which can be explained by the presence of stretch-activated cation-nonselective channels and/or load dependent factors. [3,4] Among cardiac electrophysiological modifications under stretch have been reported depolarized diastolic membrane potentials, accelerated repolarization, action potential duration shortening, refractoriness, wavelength abbreviation and altered propagation. [5]

Experimental studies analysing the effects of MEF, describing independent factors contributing to these modifications and pharmacological interventions, have been performed in different species and models of MEF, including rabbits. [6-8] However, little is known about its modifications in different layers across the myocardial wall and the time-course of MEF intramural ENDO-EPI components at fast rhythms. Understanding these modifications may require a simultaneous dual-layer approach, which is intrinsically challenging in small-tomid sized hearts. In this study, we optically mapped the EPI LV-surface and developed a MEA-balloon for ENDO mapping with volume control to set the strain and amount of STR, using a custom-developed multichannel acquisition system. Our aim was to validate the system and to study the modifications produced by moderate STR on normal and abnormal activation.

\section{Methodology}

Simultaneous ENDO-MEAb electrical and EPI-OM 
was performed in Langendorff-perfused NZW rabbit hearts $(n=10)$. A miniaturized multipurpose and extensible MEAb was developed, and controlled with a custom scalable FPGA-based system and a .NET-based user interface. After signal/image quality evaluation, ENDO and EPI restitution properties, before and after STR were studied. Additionally, components during spontaneous tachycardia and fibrillation were also explored.

\subsection{Stretching and registering device}

A miniaturized and extensible MEAb was developed with 13 unipolar electrodes equally distributed, for both continuous registration and/or stimulation. Electrodes, distributed from apex to base and introduced using the septal region as a reference, as shown in Figure 1, were constructed from stainless steel and connected by $0.005^{\prime \prime}$ thick steel insulated wire. Wires were attached into a closed cylinder-shaped expandable fabric mesh of $14 \times 4 \mathrm{~mm}$ dimensions. A nitrile expandable balloon with ellipsoidal shape was inserted into the fabric with a PVC biocompatible tube and attached to a three-way stop-cock to be able to control volume and pressure in the LV cavity. A reservoir syringe was connected in the opposite side to generate volume within the thin latex balloon.
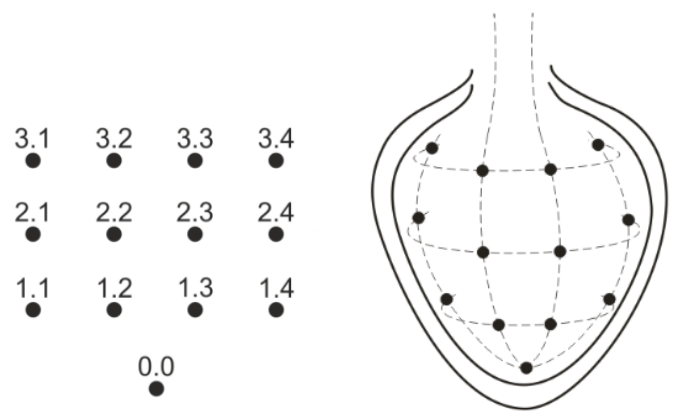

Figure 1. Mesh distribution and numbering of electrodes within the endocavitary balloon. Electrodes were uniformly distributed to cover the entire inner wall of the LV.

Increasing filled volume from physiological loading up to $1.5-2 \mathrm{ml}$ induced myocardial stretch in the LV in the latter part of the experiment.

\subsection{Setup and synchronized acquisition control}

We devised an acquisition system integrated with the perfusion circuit to maintain the heart. Control of registration was done via user interfaces developed in .NET-based and MATLAB (2017a, The MathWorks) environments. MEAb endocavitary electric recordings acquisition was obtained with a near real-time custom acquisition system based on FPGA, STM32F microcontroller and an integrated analogue front-end
(ADS1298, Texas Instruments). Recording system features were 1 to 128 simultaneous non-filtered channels, 1 to $32 \mathrm{kHz}$ selectable sampling frequency and 17 to 24-bit resolution. One $\mathrm{kHz}$ and 24-bit were configured for this work.

Continuous monitoring during the whole experiment was allowed. Registration control was done either using the scientific camera as master clock during dual-sided mapping, or via direct software intervention for singlelayer acquisition (electrical recordings). A schematic of the system is shown below (Figure 2). Stimulation was connected to specific electrode/s in the MEAb and carried out by an external GRASS S88 stimulator (Grass Instruments Inc., Quincy, MA, USA) controlling stimulation protocols with a laptop via serial port. The stimulator is equipped with a stimulus isolation unit (SIU5). Stimuli were $2 \mathrm{~ms}$ bipolar rectangular pulses with amplitude twice the diastolic threshold.

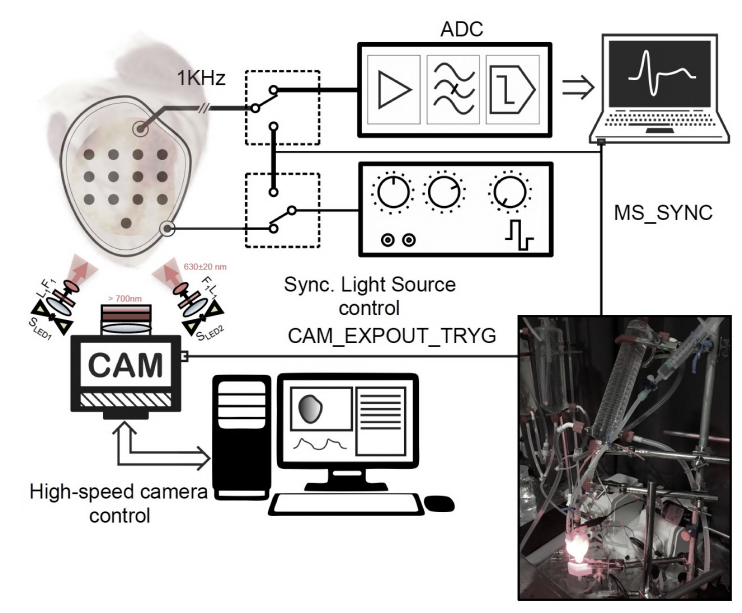

Figure 2. Schematic illustration of the acquisition system and Langendorff-perfused preparation during the experiment.

High-resolution EPI optical mapping was performed using di-4-ANBDQPQ and $7.5 \mathrm{uM}$ blebbistatine. Dual sided LED-based illumination was collimated (planoconvex spherical lenses; Thorlabs, Newton, NJ, USA), passed through excitation filters and focused on the LV epicardium. Fluorescence emission from dyeloaded myocardium was passed through one multiband emission filter and collected by the camera at $530 \mathrm{fps}$ (Evolve 128 EMCCD camera; $128 \times 128$ pixels; Photometrics, AZ, USA). Dual sided acquisition was fully synchronized. Both, electrical signals and optical sequential images, were synchronized through a monostable-based triggering circuit.

\subsection{Experimental model and protocol}

Animal care and the experimental protocols used in 
this study complied with EU directive 2010/63 on the protection of animals used for scientific purposes, and were approved by the Institutional Animal Care and Use Committee of the University of Valencia (CEEA:A1404746730665). Adult male New Zealand White rabbits $(\mathrm{n}=10)$ weighing $4.7 \pm 0.2 \mathrm{~kg}$ were used in the present study. Following pre-medication with ketamine $(35 \mathrm{mg} / \mathrm{kg}$ ) and heparinization (2500 IU), the animals were killed with an overdose of sodium pentobarbitone $(100 \mathrm{mg} / \mathrm{kg})$ administered via injection through the left marginal ear vein. Then the hearts were removed through a midsternal incision and connected to a Langendorff system for the perfusion of Tyrode solution at a pressure of $80 \mathrm{mmHg}$ and a temperature of $37 \pm 0.5^{\circ} \mathrm{C}$. The millimolar composition of the perfusion fluid was as follows: $\mathrm{NaCl} 130, \mathrm{NaHCO}_{3}$ 24.2, $\mathrm{KCl} 4.7, \mathrm{CaCl}_{2} 2.2$, $\mathrm{NaH}_{2} \mathrm{PO}_{4} 1.2, \mathrm{MgCl}_{2} 0.6$ and glucose 12. Oxygenation was carried out with a mixture of $95 \% \mathrm{O}_{2}$ and $5 \% \mathrm{CO}_{2}$.
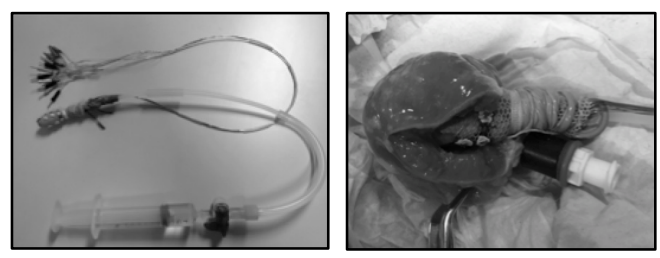

Figure 3. Developed device. Shown are views outside, and inside the LV (cut for extraction after the experiment).

An incision was made in the atrium and another in the mitral valve before inserting the balloon into the LV. An experimental protocol for evaluation of the acquisition system and further studying ENDO-EPI activation and repolarization restitution properties was devised. First, EPI stimulation was performed at specific landmarks to fine-tune the MEAb positioning, evaluate signal-to-noise (SNR) ratios, and assess consistency of endocardial breakthroughs at expected electrode positions. Second, fixed-frequency endocardial stimulation from each electrode was sequentially performed to assess patterns of activation and repolarization from each location using dual-sided EGM-OM mapping. Third, hearts were paced at specific decreasing cycle lengths to study restitution properties, from $250 \mathrm{~ms}$ to $140 \mathrm{~ms}$, to compare frequencydependent activation-repolarization components and patterns of propagation, before and after STR.

\subsection{Signal/image sequence processing and parameterization mapping}

Reconstructed action potentials and EGMs were filtered appropriately in time and space [6,9]. SNRs were obtained from the energy spectrum, as previously described. Action potential durations at $30-90 \%$ repolarization were obtained from activation and repolarization times (AT/RT) from first and second order derivatives. Activation-recovery intervals (ARI) were determined from unipolar EGMs from $\mathrm{dV} / \mathrm{dt}_{\text {min }}$ of the depolarizing slope to the positive or negative derivative on the repolarization wave slope upon its morphology. EPI APDs and ENDO ARIs were determined at each stimulation frequency, before and during STR. Repeated measures one-way ANOVA was used $(\mathrm{P}<0.05)$. Tachycardia and fibrillatory conduction was spontaneously induced in some hearts. Spatiotemporal components from each layer were compared in terms of quantified spectral and phase-domain markers (not shown).

\section{Results}

After septal-oriented insertion of the MEAb, a sequential bidirectional stimulation protocol, for reproducible and fine-tuned anatomical alignment of the endocavitary balloon, was initially done together with a subsequent camera calibration. Stimulation from both epicardium and endocardium allowed to verify breakthrough sites, maximizing inside-out spectral coherence and setting up reference anatomical positions. Table 1, highlights regional signal quality and averaged coherence values after alignment. Overall $\mathrm{SNR}_{\mathrm{EGMs}}$ was $25.69 \pm 2.85 \mathrm{~dB}$.

Table 1. Regional signal quality of electrical and optical recordings, and averaged mean spectral block-matching coherence evaluation after alignment.

\begin{tabular}{lccc}
\hline Source/Region & SNR $(\mathrm{dB})$ & Amplitude & SpCoh(A.U.) \\
\hline EGM/base & 19.28 & $2.64 \mathrm{mV}$ & 0.79 \\
EGM/mid & 24.11 & $3.15 \mathrm{mV}$ & 0.91 \\
EGM_apex & 33.68 & $2.87 \mathrm{mV}$ & 0.83 \\
OMAP_base & 49.65 & A.U. & 0.79 \\
OMAP_mid & 56.34 & A.U. & 0.91 \\
OMAP_apex & 51.42 & A.U. & 0.83 \\
\hline
\end{tabular}

Activation from either epicardium or endocardium from specific extreme positions (anterior and posterior mid lateral wall) showed consistent propagation in opposite directions.

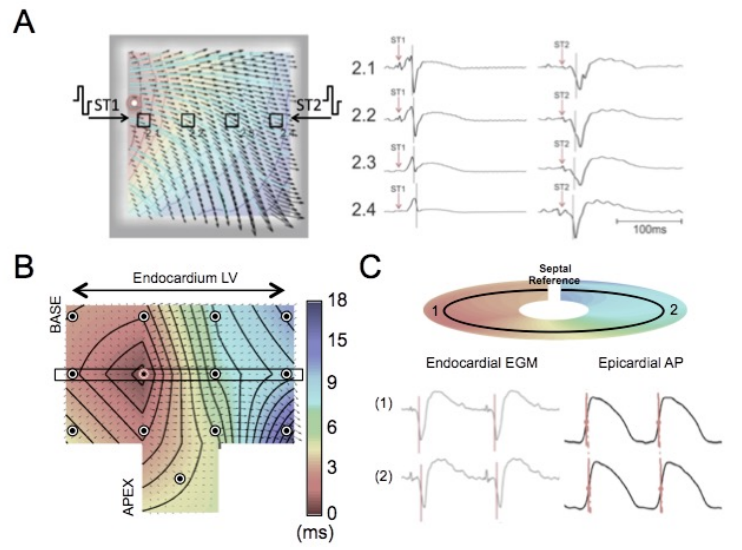

Figure 4. A. Sample recordings from endocardial activation 
sequence during epicardial stimulation from: ST1 anterior medium wall, ST2 posterior medium wall. B. Activation sequence during endocardial stimulation at $4 \mathrm{~Hz}$. C. Single-lead EGMs and single pixel APs from highlighted locations.

ARIs highly correlated with $\mathrm{APD}_{80}$, as shown in Figure 5A $\left(\mathrm{R}^{2}=0.96\right)$. Repolarization APDs and RTs were significantly larger at slower pacing cycle lengths, 250 $\mathrm{ms}$, yet tend to be shortened at faster rates such as $140 \mathrm{~ms}$ (Figure 5B).
A

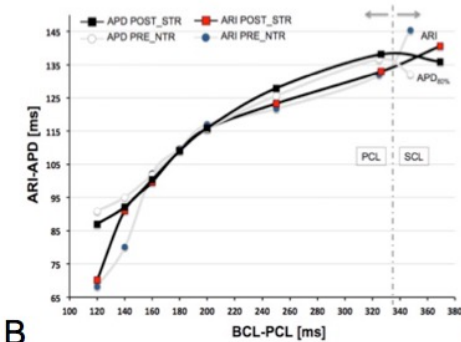

B

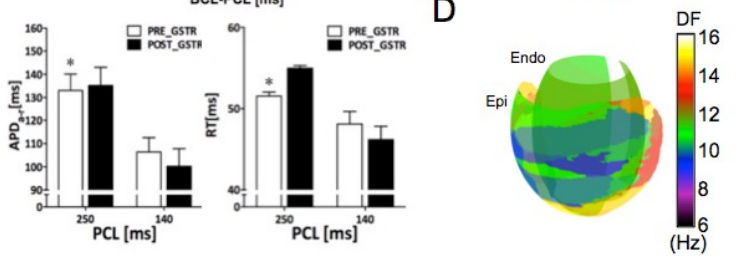

Figure 5. Modifications produced by the multielectrode array balloon on electrophysiological parameters after STR, ${ }^{*} \mathrm{p}<0.05$.

This biphasic response in activation-repolarization dynamics was consistent at different repolarization levels, with different cut-off PCLs. Arrhythmia was induced spontaneously in some hearts $(n=3)$ and accelerated under STR $(13.63 \pm 1.51$ vs $16.88 \pm 2.52 \mathrm{~Hz}, \mathrm{p}<0.05)$. Spectral components were highly correlated during regular tachycardia, observed in one heart, and dissociated during rapid fibrillatory conduction observed under STR-driven VF (Figure 5C-D).

\section{Discussion and conclusion}

We obtained simultaneous registers from inner and outer layers of the heart ventricular surface, using a dualsided approach to obtain endocardial electrical electrograms and epicardial optical transients. This method was employed to record simultaneous electrograms while modifying in a controlled manner LV volume generating myocardial STR. Under these conditions we studied differences in activationrepolarization patterns at physiological volume and under STR.

A limitation of the study is the number of electrodes considered for endocardial mapping, which may affect its correlation with high-resolution epicardial maps; yet the acquisition system developed allows for increasing the number of channels in a future prototype. Furthermore, we compared different modalities in each layer, which should be taken into consideration for interpretation of the results. Finally, we didn't study in detail intramural components during fibrillatory rhythms since it was not the objective in this preliminary validation study, thus care should be taken extrapolating the presented results.

In this study, we validated a miniaturized multielectrode array balloon for exploring simultaneously ENDO-EPI modifications during mechanoelectric feedback in rabbit hearts and a custom multichannel acquisition system for cardiac electrophysiological studies. Differences found in restitution properties could be implicated in stretch-driven VF-mechanisms, which will be compared in detail in future electrophysiological studies.

\section{Acknowledgements}

This study has been supported by research grants GV/2015/019 (Generalitat Valenciana), FIS PI15/0074801408 and CB16/11/00486 (Instituto de Salud Carlos III).

\section{References}

[1] Pfeiffer ER, et al. Biomechanics of cardiac electromechanical coupling and mechanoelectric feedback. J Biomech Eng. 2014 Feb;136(2):021007.

[2] M.J. Lab. Contraction-excitation feedback in myocardium: Physiological basis and clinical relevance. Circ Res 1982;50:757-766

[3] Orini $M$, et al. Mechano-electrical feedback in the clinical setting: Current perspectives. C. Prog. Biophys. Mol. Biol. 2017;S0079-6107(17)30040-8

[4] Lab MJ. Transient depolarization and action potential alterations following mechanical changes in isolated myocardium 1980,14,11:624-637

[5] M.R. Franz, et al. Electrophysiological effects of myocardial stretch and mechanical determinants of stretch-activated arrhythmias. Circulation 1992;86:968-978

[6] Chorro FJ, et al. Effects of myocardial stretching on excitation frequencies determined by spectral analysis during ventricular fibrillation

[7] ChorroFJ, et al. Ranolazine Attenuates the Electrophysiological Effects of Myocardial Stretch in Langendorff-Perfused Rabbit Hearts. Cardiovasc Drugs Ther. 2015 Jun;29(3):231-41.

[8] Quinn TA, Kohl P. Rabbit models of cardiac mechano-electric feedback and mechano-electrical coupling. Prog. Biophys. Mol. Biol. 2016; 121 (2):110:22

[9] Laughner JI, et al. Processing and analysis of cardiac optical mapping data obtained with potenciometric dyes. Am J. Heart Circ Physiol. 2012; 303(7):H753:H765

Address for correspondence.

Conrado J. Calvo

Innovations on Bioengineering and Electrophysiology.

Department of Electronic Engineering.

Universitat Politècnica de València.

Departament Fisiologia. Universitat de València.

conrado.calvo@uv.es 